Ponce-de-León-Elizondo, A., Sanz-Arazuri, E., Sáenz-de-Jubera-Ocón, M. y Alonso-Ruiz, R.A. (2019). La jornada lectiva a debate para (re)convertir los centros educativos en espacio de ocio. Revista de Investigación Educativa, 37(2), 395-411.

DOI: http://dx.doi.org/10.6018/rie.37.2.322751

\title{
La jornada lectiva a debate para (re)convertir los centros educativos en espacios para el ocio
}

\author{
School day to discussion, (re)converting school in spaces \\ for leisure
}

\author{
Ana Ponce-de-León-Elizondo', Eva Sanz-Arazuri, Magdalena Sáenz-de-Jubera-Ocón \\ y Rosa-Ana Alonso-Ruiz \\ Departamento de Ciencias de la Educación. Facultad de Letras y de la Educación. Universidad de La Rioja (España)
}

\begin{abstract}
Resumen
Las evidencias científicas señalan que las instalaciones educativas se configuran en espacios privilegiados para el fomento de la práctica de actividades físicas de ocio entre los jóvenes, pero ¿se constituyen en escenarios capitales para la práctica de cualquier tipo de ocio juvenil? El presente estudio indaga sobre los vínculos entre la organización de los horarios de clases de los centros educativos españoles de educación secundaria postobligatoria y el uso que hacen sus estudiantes de los espacios educativos para la práctica de ocio. Se adopta un enfoque metológico de carácter cuantitativo, desarrollado mediante la aplicación de un cuestionario elaborado ad hoc a una muestra de 1.764 estudiantes de todo el territorio español. El análisis de los datos se configura en dos fases, un análisis descriptivo y un análisis inferencial bivariado, a través de tablas de contingencia. Los resultados evidencian la predominancia de la jornada lectiva continua matutina frente a otras planificaciones de horarios de clase en la educación secundaria

1 Resultados de la investigación “De los tiempos educativos a los tiempos sociales: la cotidianidad familiar en la construcción del ocio físico-deportivo juvenil” (EDU2012-39080-C07-05) [años 2012-2015], y fundamento de la actual investigación “Ocio y bienestar en clave intergeneracional: de la cotidianidad familiar a la innovación social en las redes abuelos-nietos" (EDU2017-85642-R) [años 2017-2020]; ambas cofinanciadas en el marco del Plan Nacional I+D+I con cargo a dos ayudas del Ministerio de Economía y Competitividad, y del Fondo Europeo de Desarrollo Regional (FEDER).

Correspondencia: Ana Ponce-de-León-Elizondo, ana.ponce@unirioja.es, Universidad de La Rioja (Edificio Vives), c/ Luis de Ulloa s/n, 26004 Logroño.
\end{abstract}


postobligatoria de España, siendo, además, el horario preferido por los estudiantes. Se descarta la influencia que el tipo de jornada educativa pudiera tener en el uso del centro educativo en el tiempo de ocio de los jóvenes, mientras que sí existe relación significativa entre las preferencias de la jornada escolar y la utilización de los espacios educativos.

Palabras clave: educación; ocio; centro de enseñanza; jornada escolar.

\begin{abstract}
Scientific evidence shows that educational facilities are a privileged space to promote adolescents' practice of physical leisure activities, but do they constitute as an essential space for any kind of youth leisure? This study examines the links between the organization of class schedules in Spanish schools of post-compulsory secondary education and the use these students make of the leisure spaces. It uses a quantitative methodological approach, carried out by the application of an ad hoc developed questionnaire to a sample of 1.764 students in the entire Spanish territory. The data analysis is conducted in two stages: a descriptive analysis and a bivariate inferential analysis, by means of contingency tables. The results show the predominance of the continuous morning school day versus other class schedules in Spain's post-compulsory secondary education. Moreover, it is the students' preferred schedule. The influence of the type of school day regarding the use of the educational center during the youths' leisure time is ruled out, but there is a significant relation between school day preferences and the use of educational spaces.
\end{abstract}

Keywords: education; leisure; school; school day.

\title{
Introducción
}

El centro educativo se configura en un escenario privilegiado donde el ocio, como realidad esencial para el desarrollo personal y social desde la infancia hasta la juventud, debe constituirse en un factor fundamental de la educación integral del alumnado, por lo que estas instituciones deben asumir un compromiso pedagógico en la educación del ocio de las distintas etapas educativas (Caballo, Caride \& Gradaílle, 2012; Ferreira, Pose \& De Valenzuela, 2015) y en la promoción de la salud y el bienestar de los estudiantes (Dewhirst et al, 2014; Sansanwal, Derevensky, Lupu, \& Lupu, 2015).

El grueso de las investigaciones que abordan el tándem ocio-escuela se han centrado, principalmente, en las etapas de escolaridad obligatoria (Caballo et al., 2012; Morán \& Varela, 2011), de modo que el grado de estudios que tratan la influencia que ejerce el centro educativo se desvanece en etapas postobligatorias, si bien existen estudios, como el realizado por Valdemoros, Sáenz-de-Jubera y Alonso (2017), que ponen de manifiesto que el profesorado de educación secundaria postobligatoria opina, en su mayoría, que los centros educativos tienen una gran responsabilidad en la educación del ocio para construir experiencias activas y saludables.

El presente estudio pretende seguir profundizando en esta etapa educativa; de entre todos los escenarios donde el alumnado practica actividades de ocio, caso de locales privados, asociaciones, clubes, sociedades u otros espacios, como centros comerciales o cines, se destaca al centro educativo como lugar esencial, dado su compromiso social en la educación del alumnado. 
Las instalaciones de los centros educativos son una parte fundamental de la red de equipamientos de las comunidades autónomas. Esta afirmación se ve reforzada por los hallazgos de distintas investigaciones que constatan que una de las razones que desmotivan la práctica de experiencias específicas de ocio se vincula con la ausencia de instalaciones apropiadas accesibles para los jóvenes (Gálvez, 2004; Sanz, 2005). La lejanía a las instalaciones se establece en causa principal de la falta de práctica; estudios como los Chambers et al. (2017) constatan que los adolescentes y jóvenes destinan la mitad de su tiempo de ocio en su barrio. Sin embargo, en este estudio también se destaca que las propuestas de ocio en el centro educativo es lo que, prioritariamente, les mueve a salir del barrio.

Si bien, las instalaciones de los centros educativos, se constituyen en espacios esenciales para el fomento de hábitos físico-deportivos entre los escolares (Viciana \& Mayorga-Vega, 2017), autores como Ramos, Ponce-de-León y Sanz (2010) corroboran que son de las menos utilizadas para la realización de actividades físico-deportivas de ocio, ahora bien, ¿ocurre lo mismo con otros tipos de ocio?

Estudios previos ponen de manifiesto la necesidad de que los centros dispongan de infraestructuras actualizadas y de equipamiento adecuado (Herrador, 2006) que faciliten la adhesión a las experiencias de ocio (Gil, Felipe, Burillo, García-Tascón \& Gallardo, 2010), y sean utilizadas fuera del horario lectivo y favorezcan una mayor autonomía y autogestión del ocio en la juventud (Ortega, Lazcano \& Manuel, 2015; Sanz \& Ponce-de-León, 2014).

En esta línea, las distintas comunidades autónomas apuestan por que los centros educativos permanezcan abiertos en horario vespertino para el uso de sus instalaciones deportivas y recreativas, con un uso libre o dirigido, en tanto en cuanto los centros ofertan amplios programas de actividades extraescolares y de ocio que facilitan la permanencia de los estudiantes durante toda la jornada en el centro educativo, principalmente por la importancia de ocupar el tiempo fuera del horario lectivo en prácticas de carácter educativo, creativo o lúdico (Argüelles, Campos \& López, 2015; Ricoy \& Fernández-Rodríguez, 2016).

Las razones temporales, entre las que se incluyen los tiempos escolares, pueden constituirse en un elemento a tener en cuenta para acceder o mantener la práctica de actividades de ocio en las instalaciones educativas, afectando esta cuestión a su práctica continuada, revelándose que los horarios son, para un elevado porcentaje de jóvenes, un motivo de abandono de sus experiencias de ocio (Caballo, Varela \& Nájera, 2017; Gálvez, 2004; Madariaga \& Romero, 2016; Sanz, Valdemoros, Ramos \& Ponce-de-León, 2011). Asimismo, los hallazgos de Cruz y Morán (2016) señalan que los estudiantes de educación secundaria postobligatoria muestran una preferencia mayoritaria por la modalidad de jornada continua matutina; por su parte las investigaciones de Fraguela, Lorenzo, Merelas y Varela (2013) revelan que las familias que manifiestan estar menos satisfechas con los horarios escolares de los hijos e hijas, independientemente del tipo de jornada, son aquellas que peores niveles de conciliación experimentan, aseverando que estos tiempos educativos determinan su organización familiar cotidiana. Todas estas constataciones hacen necesario profundizar en torno a la modalidad organizativa de la jornada lectiva de los centros educativos, por ser susceptible de condicionar la práctica de ocio de estos jóvenes en los espacios educativos y el funcionamiento familiar. 
En referencia a este importante agente educativo, distintos estudios formalizados en las últimas tres décadas, muestran una tendencia al alza respecto a la participación de la familia en el centro educativo, si bien dista aún de los niveles óptimos de colaboración, máxime, si se tienen en cuenta los numerosos beneficios que produce en los estudiantes y sus familiares, tales como la mejora de la cohesión social, la inclusión o el rendimiento académico, así como las expectativas de profesores y progenitores en lo referente al proceso educativo (Colás \& Contreras, 2013; Santos, Lorenzo \& Priegue, 2011; Sarramona \& Roca, 2007). Cuestión que invita a la reflexión de la necesidad de implicación y participación de las familias en la gestión de los tiempos en el centro escolar.

Si bien la literatura científica apenas se ha hecho eco de los tiempos educativos, sí ha abordado las bondades e inconvenientes de la jornada lectiva partida (se desarrolla en sesiones de mañana y tarde) y la continua (tiene lugar en sesiones, generalmente, de mañana, pero también de tarde) siendo, a su vez, objeto de numerosos debates entre los miembros de la comunidad educativa, esgrimiendo fundamentalmente razones pedagógicas, de convivencia, laborales y familiares para posicionarse a favor de una u otra modalidad. Así, distintos autores concluyen que la jornada partida frente a la jornada continua, conlleva una reducción de la permanencia del alumnado en la institución educativa, merma en la calidad de las actividades extraescolares ofertadas, propicia la falta de aprovechamiento de los recursos de las instituciones educativas o la eliminación de actividades deportivas o talleres, además de que contribuye al incremento de desigualdades sociales ante la educación (Morales, 2013; Tinajas, 2012).

A partir de las premisas citadas y de la escasez de investigaciones sobre la temática abordada se lleva a cabo el presente estudio que, a su vez, se inserta dentro de un proyecto coordinado mucho más ambicioso en el que se analizan en profundidad los tiempos educativos y sociales de los estudiantes de educación secundaria postobligatoria, con el fin de identificar problemáticas y alternativas pedagógico-sociales que favorezcan una construcción valiosa de la condición juvenil.

\section{Método}

\section{Objetivos}

El objetivo de este estudio trata de identificar vínculos entre la organización de los horarios de clases de los centros educativos españoles de educación secundaria postobligatoria y el uso que hacen sus estudiantes de los espacios educativos para la práctica de ocio.

\section{Población y Muestra}

La población objeto de estudio estuvo conformada por los estudiantes de educación secundaria postobligatoria de régimen general, matriculados en centros educativos del Estado Español. Según datos de estadística de las enseñanzas no universitarias de la Subdirección General de Estadística y Estudios del Ministerio de Educación, Cultura y Deporte, este universo de trabajo ascendía, en el momento de realización del trabajo de campo, a 1.055.532 estudiantes. 
Para esta población finita, el tamaño muestral necesario debía ascender a 1.813 alumnos para un error de muestreo de $2.3 \%$ y un nivel de confianza del $95 \%$.

Finalmente, tras la recogida y validación de los datos se registró una mortalidad experimental del $2.70 \%$, por lo que la muestra final quedó conformada por un total de 1.764 estudiantes. El $50.2 \%$ mujeres $(n=885)$ y el $49.8 \%$ hombres $(n=879)$; la media de edad fue de $17.60 \pm 1.60$, un $57.1 \%(n=1007)$ de los participantes contaban en el momento del estudio con 18 años o menos y un 42.9\% $(n=775)$ con 19 años o más; un $63.5 \%(n=1120)$ fueron estudiantes de Bachillerato, un $27.9 \%(n=492)$ de Ciclo Formativo de Grado Medio y un 8.7\% $(\mathrm{n}=153)$ de Programas de Capacitación Profesional Inicial -PCPI-; el 76.7\% (n=1352) pertenecía a centros públicos y el $23.3 \%(n=$ 412) a privados. El tipo de muestreo fue aleatorio simple con afijación proporcional, atendiendo a los siguientes criterios: áreas del territorio español -noreste, levante, sur, centro, noroeste y norte- (tabla 1), titularidad del centro (públicos y privados), tipo de estudios (Bachillerato, Formación Profesional de Grado Medio y Programas de Capacitación Profesional Inicial -PCPI-) y sexo (hombre o mujer).

Tabla 1

Agrupación territorial para la muestra

\begin{tabular}{llccc}
\hline $\begin{array}{l}\text { Denominación/ } \\
\text { Localización }\end{array}$ & Comunidades y/o provincias & Población & $\begin{array}{c}\text { Muestra } \\
\text { prevista }\end{array}$ & $\begin{array}{l}\text { Muestra } \\
\text { real }\end{array}$ \\
\hline A1-Noreste & $\begin{array}{l}\text { Cataluña, Aragón (excepto Teru- } \\
\text { el) y Baleares }\end{array}$ & 197.709 & 340 & 311 \\
A2-Levante & $\begin{array}{l}\text { Comunidad Valenciana, Murcia } \\
\text { y Albacete }\end{array}$ & 166.674 & 286 & 286 \\
A3-Sur & $\begin{array}{l}\text { Andalucía, Islas Canarias, Ceuta } \\
\text { y Melilla }\end{array}$ & 283.247 & 486 & 466 \\
A4-Centro & $\begin{array}{l}\text { Madrid, Castilla-La Mancha } \\
\text { (excepto Albacete), Castilla-León } \\
\text { (excepto León, Palencia y Bur- } \\
\text { gos), Cáceres y Teruel }\end{array}$ & 227.564 & 391 & 391 \\
& $\begin{array}{l}\text { Galicia, Asturias y León } \\
\text { Cantabria, País Vasco, La Rioja, }\end{array}$ & 91.753 & 158 & 152 \\
A5-Noroeste & Navarra, Burgos y Palencia & & & \\
A6-Norte & & & & \\
\hline
\end{tabular}

Nota: elaboración propia a partir de las áreas delimitadas por el instituto de investigación de mercados Nielsen

\section{Instrumento}

Se elaboró un cuestionario ad hoc, con 44 preguntas, para conocer la organización de los tiempos educativos y sociales de los estudiantes españoles de Educación Secundaria Postobligatoria. Para la validación del instrumento se desarrolló una prueba piloto en ocho comunidades autónomas en la que participaron 248 estudiantes de diferentes tipos de estudios de educación secundaria postobligatoria, con el fin de 


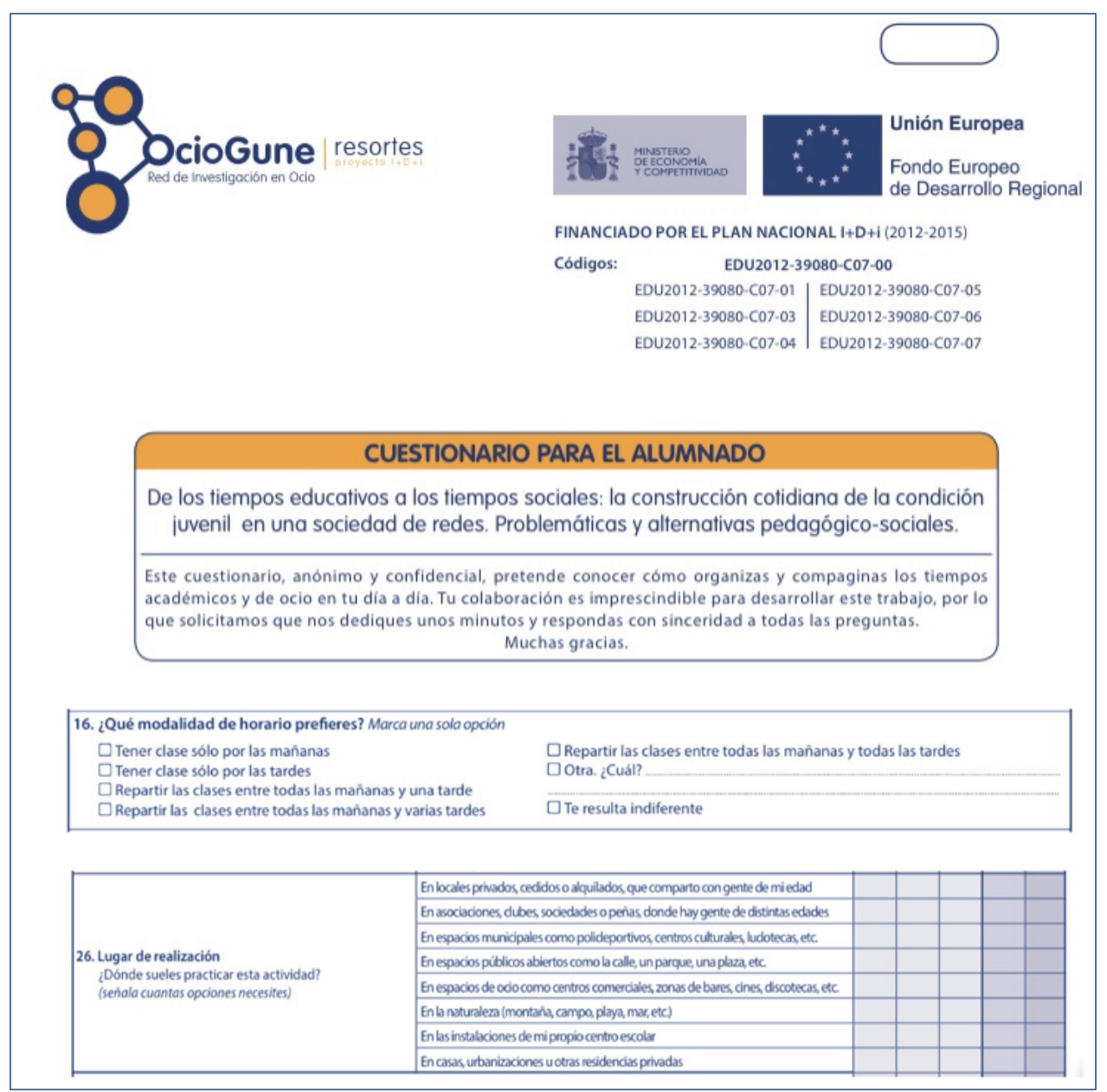

Figura 1. Extracto de los ítems empleados del instrumento de medida

detectar errores de interpretación que guiaran la depuración de la redacción de las preguntas. Tras las correcciones oportunas surgidas del análisis de la prueba piloto, la nueva versión del cuestionario fue sometida al juicio de 14 expertos pertenecientes a siete universidades españolas. Estos debieron medir, a través de una escala Likert de cinco puntos y para cada ítem, la pertinencia con el objetivo a medir, la no influencia de la redacción en la respuesta, la claridad en el lenguaje empleado, la adecuación del lenguaje a los encuestados y la inclusión de todas las alternativas posibles. El juicio de expertos determinó que cada uno de los ítems del cuestionario permitía recoger claramente la información deseada para cada variable del estudio, superando en todos los casos una puntuación media de 4 puntos. Las pequeñas matizaciones aportadas por los expertos en el apartado de observaciones permitieron aumentar la calidad de la versión definitiva. 
En el presente trabajo se analizan los datos acopiados en torno a tres variables recogidas por los ítems 16 y 26 del instrumento descrito:

Horario de clases del centro: los investigadores registraron, para cada centro participante en la muestra, los horarios de clase, estableciendo seis posibles categorías: solo clases por la mañana, solo clases por la tarde, clases todas las mañanas y una tarde, clases todas las mañanas y varias tardes, clases todas las mañanas y todas las tardes y, por último, otra opción.

Modalidad de horario preferido por el estudiante: los jóvenes indicaron su preferencia de horarios de clase entre seis categorías coincidentes con las de la variable anterior.

Utilización de los espacios del centro educativo para practicar sus actividades de ocio principales: cada estudiante indicó si practicaba cada una de sus actividades principales de ocio en las instalaciones del centro. Esta variable es dicotómica con dos posibles opciones (sí/no).

Se hace necesario aclarar, en este punto, que por actividades de ocio se entiende cualquier actividad que la persona decide libremente para invertir su tiempo libre de obligaciones (laborales, educativas, familiares y fisiológicas), que le produce satisfacción, así como desarrollo personal y social. Bajo esta concepción, para el presente estudio, se han contemplado nueve tipos de actividades de ocio: televisión y radio, ocio digital, deporte y actividad física, turismo y excursionismo, actividades culturales, fiestas/ celebraciones, juegos de mesa, asociacionismo y voluntariado y otras actividades.

\section{Procedimiento de recogida y análisis de datos}

La aplicación del cuestionario se desarrolló cumpliendo fielmente con el total de encuestas previstas en cada una de las seis áreas territoriales, sin necesidad de visitar todas las provincias incluidas. Antes de proceder a la aplicación del instrumento se solicitó permiso al Director General de Educación de cada Gobierno Autonómico. Una vez obtenido el permiso, se contactó con los directores de diversos centros educativos de cada Comunidad Autónoma para, tras informarles sobre los objetivos del estudio, solicitar la posible participación de algunos de sus alumnos en la cumplimentación del cuestionario. Atendiendo a una distribución por conglomerados, se seleccionaron los centros educativos participantes de entre aquellos de los que se obtuvo respuesta positiva y cumplían con los criterios de la configuración de la muestra. Para aplicar los cuestionarios, dos investigadores acudieron presencialmente a cada uno de los centros de educación secundaria postobligatoria seleccionados, adjuntando una carta informativa dirigida a las familias de los estudiantes encuestados. En cada centro se aplicó el instrumento en el número de aulas estimado como necesario para cumplir con las cuotas, suponiendo una media de 25 alumnos por clase. Los participantes cumplimentaban el cuestionario durante una hora lectiva dentro de su clase-aula y bajo la supervisión constante de los investigadores, quienes les informaron de los objetivos de la investigación, de la ética de la investigación garantizando el carácter voluntario y la protección de derechos y garantías de los participantes, conservando en todo momento el anonimato y la confidencialidad de los datos aportados.

Los resultados registrados se han tratado y analizado con ayuda del paquete estadístico SPSS 25.0. En primer lugar, se efectuó un análisis descriptivo de las variables objeto de este 
estudio, indicando la frecuencia de cada una de las categorías estudiadas. En un segundo paso, se llevó a cabo un análisis inferencial bivariado, a través de tablas de contingencia y empleando el coeficiente $\mathrm{V}$ de Cramer para dar respuesta a las siguientes cuestiones:

- ¿Los horarios de clase se asocian con un mayor uso de los espacios educativos para la práctica de ocio?

- ¿Los jóvenes que practican ocio en las instalaciones del centro educativo muestran mayor preferencia por algunas modalidades de horario frente a otras?

Dado que el valor del coeficiente $\mathrm{V}$ de Cramer oscila entre 0 y 1 , se establecieron cinco intervalos de graduación que definieron la fuerza de la asociación. De este modo, los valores entre 0.000 y 0.200 reflejaron una asociación muy débil; la horquilla entre 0.200 y 0.400 se interpretó con una relación débil; una vinculación moderada vino definida por cifras entre 0.400 y 0.600 mientras que los intervalos entre 0.600 y 0.800 y entre 0.800 y 1 se identificaron con asociaciones fuertes y muy fuertes respectivamente.

En todo el estudio se consideró un nivel de significatividad de $p$ inferior a.05.

\section{Resultados}

Respecto a la distribución horaria de clases, tres de cada cuatro alumnos manifiestan contar con un horario educativo exclusivamente de mañana; uno de cada diez declara tener clase todas las mañanas y todas las tardes y un $7.9 \%$ todas las mañanas y varias tardes. Valores residuales inferiores a 3\% se registran en las otras categorías estudiadas (figura 1).

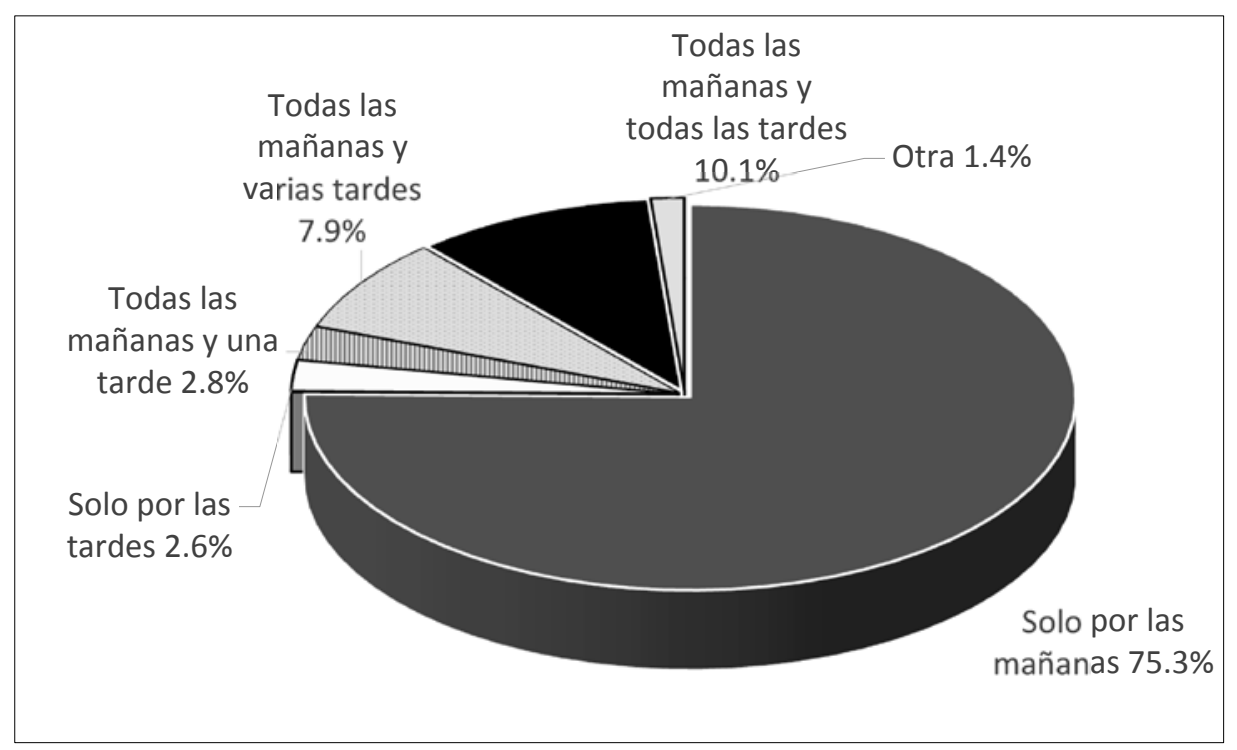

Figura 2. Horario de clases 
Al preguntar a los estudiantes qué horario lectivo preferirían, independientemente de su horario actual, se registra un $85.9 \%$ de respuestas en la categoría de solo por las mañanas, mientras que tan solo un $1 \%$ manifiesta su predilección por la jornada partida todos los días convirtiéndose claramente en la distribución menos deseada por los estudiantes. Resulta interesante destacar que un 3.4\% de los encuestados se muestra indiferente ante el horario de clases (figura 3).

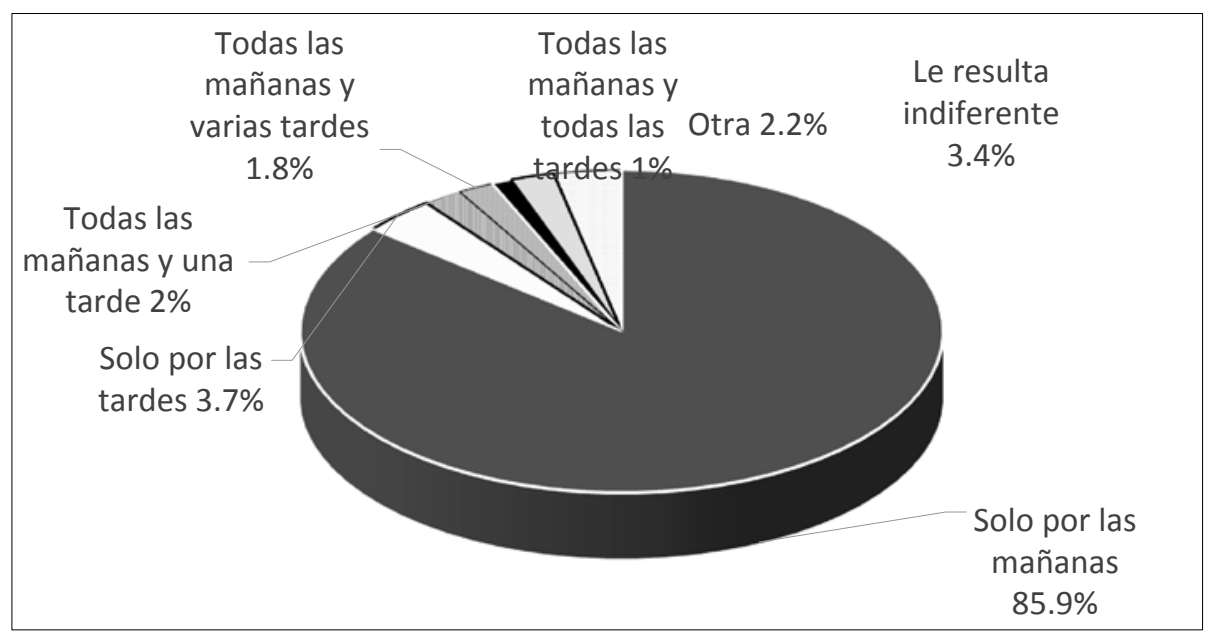

Figura 3. Horario de clases preferidos por los estudiantes

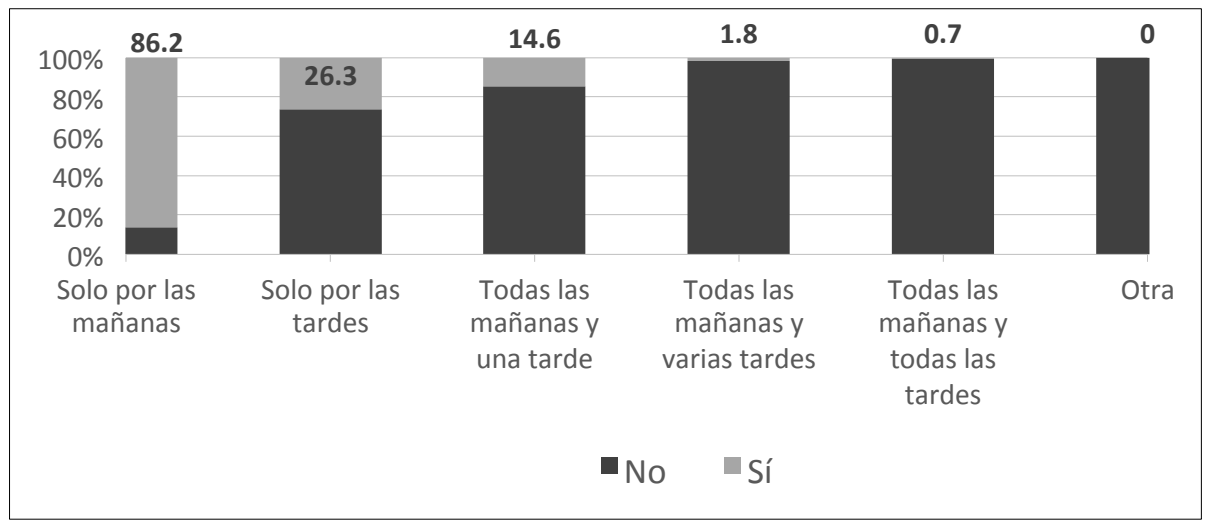

Figura 4. Jornada lectiva acorde con la preferencia horaria del estudiante

Si se cruzan estas dos variables, horario lectivo que disfrutan y jornada de clases que desearían tener, se descubre que el 58.2\% de los estudiantes de secundaria postobligatoria tienen una jornada lectiva acorde con su preferencia horaria, mientras que un $41.8 \%$ muestra preferencia por un horario lectivo diferente del que ha de cumplir. Al estudiar este aspecto segmentando a los participantes por el tipo de horarios de 
clase que siguen, se identifica que el $86.2 \%$ de los que tienen jornada lectiva de mañana desean ese horario. En el resto de jornadas lectivas el porcentaje de estudiantes que prefieren el mismo horario que han de cumplir es inferior al 30\% (figura 4).

Con el pleno convencimiento de que los espacios de los centros educativos son puntos de oportunidad también para los tiempos sociales no curriculares, este estudio indaga sobre el uso por parte de los estudiantes de su centro escolar para el disfrute de sus actividades de ocio, descubriendo que tan solo el 6.7\% de las actividades principales de ocio de los estudiantes de secundaria postobligatoria se desarrollan en las instalaciones de los centros educativos y un 15\% de los jóvenes encuestados indica practicar alguna de sus tres actividades principales de ocio en el centro en el que estudia.

\section{Relación entre el tipo de jornada lectiva y uso del centro educativo para la práctica de ocio}

El coeficiente V de Cramer muestra la inexistencia de asociación significativa entre el tipo de jornada lectiva y el uso del centro educativo para la práctica de ocio (V de Cramer $=.064 ; p>.05$ ), lo que descarta que la jornada lectiva sea un condicionante de una mayor o menor actividad de ocio en los centros de educación secundaria postobligatoria (tabla 2).

Tabla 2

Tabla de contingencia del uso del centro educativo para la práctica de ocio en función de la jornada lectiva

\begin{tabular}{|c|c|c|c|c|}
\hline \multicolumn{5}{|c|}{ Uso del centro educativo para la práctica de ocio } \\
\hline & & $\begin{array}{c}\text { No } \\
(n=1499)\end{array}$ & $\begin{array}{c}\text { Sí } \\
(n=265)\end{array}$ & Sig \\
\hline \multirow{6}{*}{$\begin{array}{l}\text { Tipo de jornada } \\
\text { educativa }\end{array}$} & $\begin{array}{l}\text { Solo por las mañanas } \\
(\mathrm{n}=1105)\end{array}$ & $\begin{array}{c}940 \\
(85.1 \%)\end{array}$ & $\begin{array}{c}165 \\
(14.9 \%)\end{array}$ & \multirow{6}{*}{$\begin{array}{c}\text { V de Cramer }=.036 \\
\qquad p=.898\end{array}$} \\
\hline & $\begin{array}{l}\text { Solo por las tardes } \\
(\mathrm{n}=38)\end{array}$ & $\begin{array}{c}31 \\
(81.6 \%)\end{array}$ & $\begin{array}{c}7 \\
18.4 \%)\end{array}$ & \\
\hline & $\begin{array}{l}\text { Todas las mañanas y } \\
\text { una tarde }(n=41)\end{array}$ & $\begin{array}{c}37 \\
(90.2 \%)\end{array}$ & $\begin{array}{c}4 \\
(9.8 \%)\end{array}$ & \\
\hline & $\begin{array}{l}\text { Todas las mañanas y } \\
\text { varias tardes } \\
(\mathrm{n}=116)\end{array}$ & $\begin{array}{c}96 \\
(82.8 \%)\end{array}$ & $\begin{array}{c}20 \\
(17.2 \%)\end{array}$ & \\
\hline & $\begin{array}{l}\text { Todas las mañanas y } \\
\text { todas las tardes } \\
(\mathrm{n}=148)\end{array}$ & $\begin{array}{c}127 \\
(85.8 \%)\end{array}$ & $\begin{array}{c}21 \\
(14.2 \%)\end{array}$ & \\
\hline & Otros $(n=20)$ & $\begin{array}{c}18 \\
(90 \%)\end{array}$ & $\begin{array}{c}2 \\
(10 \%)\end{array}$ & \\
\hline
\end{tabular}




\section{Relación entre el tipo de jornada lectiva preferida por los estudiantes y uso del centro educativo para la práctica de ocio}

Si bien el horario de clases que siguen los estudiantes de secundaria postobligatoria no se asocia significativamente al uso del centro educativo para la práctica de ocio, el análisis bivariado sí muestra diferencias significativas, aunque muy débiles, en el uso del centro educativo para las experiencias de ocio de los jóvenes en función del tipo de jornada lectiva preferida por los estudiantes (V de Cramer $=.105 ; p<.005$ ). Quienes prefieren una jornada partida con clases todas las mañanas y todas las tardes (28.6\%), junto con aquellos que desean otros tipos de jornadas diferentes a las categorizadas $(28.9 \%)$, son los que más practican actividades de ocio en el centro. En el lado opuesto, el porcentaje más bajo de usuarios de ocio en los espacios educativos se encuentra entre los jóvenes que muestran una preferencia por la jornada intensiva con clases solo por las mañanas, donde tan solo se detecta un 13.7\% de alumnos que declaran experimentar alguna actividad de ocio en el centro educativo (tabla 3).

\section{Tabla 3}

Tabla de contingencia del uso del centro educativo para la práctica de ocio en función de la jornada lectiva preferida

\begin{tabular}{|c|c|c|c|c|}
\hline \multicolumn{5}{|c|}{ Uso del centro educativo para la práctica de ocio } \\
\hline & & $\begin{array}{c}\text { No } \\
(n=1499)\end{array}$ & $\begin{array}{c}\text { Sí } \\
(n=265)\end{array}$ & Sig \\
\hline \multirow{7}{*}{$\begin{array}{l}\text { Tipo de jorna- } \\
\text { da educativa } \\
\text { preferida }\end{array}$} & $\begin{array}{l}\text { Solo por las maña- } \\
\text { nas }(n=1500)\end{array}$ & $\begin{array}{c}1295 \\
(86.3 \%)\end{array}$ & $\begin{array}{c}205 \\
(13.7 \%)\end{array}$ & \multirow{7}{*}{$\begin{array}{c}\text { V de Cramer }=.105 \\
p=.004\end{array}$} \\
\hline & $\begin{array}{l}\text { Solo por las tardes } \\
(\mathrm{n}=64)\end{array}$ & $\begin{array}{c}49 \\
(76.6 \%)\end{array}$ & $\begin{array}{c}15 \\
(23.4 \%)\end{array}$ & \\
\hline & $\begin{array}{l}\text { Todas las mañanas } \\
\text { y una tarde }(n=35)\end{array}$ & $\begin{array}{c}25 \\
(71.4 \%)\end{array}$ & $\begin{array}{c}10 \\
(28.6 \%)\end{array}$ & \\
\hline & $\begin{array}{l}\text { Todas las mañanas } \\
\text { y varias tardes } \\
(\mathrm{n}=32)\end{array}$ & $\begin{array}{c}26 \\
(81.3 \%)\end{array}$ & $\begin{array}{c}6 \\
(18.8 \%)\end{array}$ & \\
\hline & $\begin{array}{l}\text { Todas las mañanas } \\
\text { y todas las tardes } \\
(\mathrm{n}=18)\end{array}$ & $\begin{array}{c}13 \\
(72.2 \%)\end{array}$ & $\begin{array}{c}5 \\
(27.8 \%)\end{array}$ & \\
\hline & $\begin{array}{l}\text { Le resulta indife- } \\
\text { rente }(n=60)\end{array}$ & $\begin{array}{c}50 \\
(83.3 \%)\end{array}$ & $\begin{array}{c}10 \\
(16.7 \%)\end{array}$ & \\
\hline & Otros $(n=38)$ & $\begin{array}{c}27 \\
(71.1 \%)\end{array}$ & $\begin{array}{c}11 \\
(28.9 \%)\end{array}$ & \\
\hline
\end{tabular}




\section{Relación entre preferir el mismo tipo de jornada lectiva que rige sus estudios y uso del centro educativo para la práctica de ocio}

Entre el colectivo de estudiantes que tienen clases solo por la mañana, a la hora de utilizar los espacios del centro educativo para la práctica de ocio, existen diferencias significativas débiles entre quienes su preferencia horaria coincide con la que tienen y entre aquellos que muestran otra predilección (V de Cramer=.235; $p$ <.005). Los jóvenes que dicen preferir el horario intensivo de mañana practican significativamente menos ocio en el centro que quienes muestran otra preferencia diferente a la de los horarios de su plan de estudios. Entre los estudiantes que van a clase por las mañanas y además muestran su predilección por este horario, se identifica a un $13.1 \%$ de jóvenes que practican actividades de ocio en los espacios del centro educativo. Este porcentaje asciende hasta el $25.1 \%$ entre quienes yendo a clases por la mañana muestran otra preferencia horaria.

En el resto de jornadas no se detectan diferencias significativas, en cuanto al uso de estos espacios en tiempos extracurriculares, en función de si prefieren o no la misma distribución horaria para las obligaciones curriculares (tabla 4).

Tabla 4

Tabla de contingencia del uso del centro educativo para la práctica de ocio en función de si la jornada lectiva preferida coincide con la real

\section{Uso del centro educativo para la práctica de ocio}

\begin{tabular}{|c|c|c|c|c|}
\hline $\begin{array}{l}\text { Jornada lectiva } \\
\text { real }\end{array}$ & $\begin{array}{l}\text { Jornada lectiva } \\
\text { preferida }\end{array}$ & No & Sí & Sig \\
\hline $\begin{array}{l}\text { Solo por las } \\
\text { mañanas }\end{array}$ & $\begin{array}{l}\text { Solo por las mañanas } \\
\text { Otros }\end{array}$ & $\begin{array}{l}820(86.9 \%) \\
113(74.9 \%)\end{array}$ & $\begin{array}{l}124(13.1 \%) \\
38(25.1 \%)\end{array}$ & $\begin{array}{l}\text { V de Cramer }=.235 \\
p=.003^{* *}\end{array}$ \\
\hline $\begin{array}{l}\text { Solo por las } \\
\text { tardes }\end{array}$ & $\begin{array}{l}\text { Solo por las tardes } \\
\text { Otros }\end{array}$ & $\begin{array}{c}7(70 \%) \\
24(85.7 \%)\end{array}$ & $\begin{array}{l}3(30 \%) \\
4(14.3 \%)\end{array}$ & $\begin{array}{l}\text { V de Cramer }=.296 \\
p=.648\end{array}$ \\
\hline $\begin{array}{l}\text { Todas las maña- } \\
\text { nas y una tarde }\end{array}$ & $\begin{array}{l}\text { Todas las mañanas y } \\
\text { una tarde } \\
\text { Otros }\end{array}$ & $\begin{array}{l}5(83.3 \%) \\
32(91.4 \%)\end{array}$ & $\begin{array}{l}1(16.7 \%) \\
3(8.6 \%)\end{array}$ & $\begin{array}{l}\text { V de Cramer }=.117 \\
p=.906\end{array}$ \\
\hline $\begin{array}{l}\text { Todas las ma- } \\
\text { ñanas y varias } \\
\text { tardes }\end{array}$ & $\begin{array}{l}\text { Todas las mañanas y } \\
\text { varias tardes } \\
\text { Otros }\end{array}$ & $\begin{array}{l}2(100 \%) \\
92(82.1 \%)\end{array}$ & $\begin{array}{l}0(0 \%) \\
20(17.9 \%)\end{array}$ & $\begin{array}{l}\text { V de Cramer }=.143 \\
p=.802\end{array}$ \\
\hline $\begin{array}{l}\text { Todas las maña- } \\
\text { nas y todas las } \\
\text { tardes }\end{array}$ & $\begin{array}{l}\text { Todas las mañanas y } \\
\text { tardes } \\
\text { Otros }\end{array}$ & $\begin{array}{c}0(0 \%) \\
125(86.2 \%)\end{array}$ & $\begin{array}{l}1(100 \%) \\
20(13.8 \%)\end{array}$ & $\begin{array}{l}\text { V de Cramer }=.274 \\
p=.091\end{array}$ \\
\hline Otra & $\begin{array}{l}\text { La misma } \\
\text { Otros }\end{array}$ & $\begin{array}{c}0(0 \%) \\
18(90 \%)\end{array}$ & $\begin{array}{l}0(0 \%) \\
2(10 \%)\end{array}$ & $\begin{array}{l}\text { V de Cramer }=.167 \\
p=.757\end{array}$ \\
\hline
\end{tabular}




\section{Discusión y conclusiones}

Esta investigación revela la predominancia absoluta, en la educación secundaria postobligatoria de España, de la jornada lectiva continua matutina frente a otras planificaciones de horarios de clase. Tan solo uno de cada cinco estudiantes tiene una jornada lectiva partida.

Resulta llamativo que cuatro de cada diez alumnos de educación secundaria postobligatoria deseen una organización de horarios lectivos diferente a la que tienen. Un dato tan elevado de divergencia reclama la necesidad de seguir investigando sobre este aspecto, tratando de descubrir claves que favorezcan la aproximación de la oferta y la demanda horaria de los estudios de secundaria postobligatoria. Esta discrepancia es muy superior entre quienes cuentan con clases por las tardes, aumentando todavía más entre los que asisten a jornadas partidas. Estos hallazgos han de provocar la reflexión en el seno de los equipos directivos en su desempeño profesional, puesto que la planificación de los tiempos escolares es un factor fundamental en la organización de los centros educativos, que ha de buscar tanto la mejora de la calidad educativa como la conciliación de los distintos factores sociales (Caride, 2005; Morán, 2012).

Estudios como los de Cruz y Morán (2016) muestran que casi nueve de cada diez jóvenes de educación secundaria postobligatoria desean jornada intensiva solo de mañana. La presente investigación, partiendo de estos datos, profundiza en este colectivo, revelando que en seis de cada diez encuestados, coincide su preferencia matutina con el horario cursado, mientras que tres de cada diez disponen de otra jornada, a pesar de desear el turno matinal. Por otro lado, casi 4 de cada 100 estudiantes de educación secundaria postobligatoria desea turno de clases exclusivamente de tarde, pero solo uno de cada doscientos disfruta de su preferencia vespertina y seis de cada doscientos estudiantes desearía este horario, pero tiene otro. El resto de tipos de jornadas lectivas son poco deseadas por los jóvenes.

En cuanto a los tiempos extracurriculares, el presente estudio constata que las instalaciones educativas, a pesar de su idoneidad, son de las menos utilizadas por los jóvenes para la realización de actividades de ocio. Este hallazgo se sitúa en sintonía con la investigación de Ramos et al. (2010), la cual ya puso en evidencia el escaso uso de los centros educativos para la práctica de la actividad física de ocio por parte de estudiantes, en ese caso, de educación secundaria obligatoria.

La presente investigación descarta la influencia del tipo de jornada educativa en el uso del centro educativo para la práctica de ocio de los estudiantes españoles de educación secundaria postobligatoria. Estos datos contradicen las afirmaciones de Morales (2013) quien argumenta que el cambio de jornada de mañana y tarde a jornada de mañana, trae consigo una merma de la presencia de los estudiantes en las instituciones educativas. Si bien se hace necesario destacar que estas discrepancias científicas pudieran ser debidas a las diferentes etapas educativas contempladas por los autores, Morales (2013) analiza la etapa de educación primaria mientras que el presente estudio se centra en la etapa de educación secundaria postobligatoria. Esta cuestión señala la importancia de un estudio comparado en función de la variable etapa educativa en la comprensión de las preferencias, y como consecuencia oportunidades, del uso de espacios en tiempos de ocio por los estudiantes de las diferentes etapas educativas. 
Si bien el tipo de jornada no influye en que se practique más o menos ocio en los centros españoles de educación secundaria postobligatoria, el presente estudio revela que sí existe un vínculo con las preferencias de los estudiantes respecto al tipo de jornada lectiva, lo que lleva a reflexionar si los factores influyentes en el uso de los espacios educativos para la práctica de ocio tienen que ver más con motivaciones intrínsecas de los estudiantes, que con cuestiones organizativas del propio centro. No obstante, desde las políticas de organización y gestión de las instituciones educativas se pueden abordar estrategias organizativas que atiendan a las necesidades, preferencias e intereses de los jóvenes, dándoles la oportunidad de expresar sus rasgos de identidad generando, así, espacios donde alcancen mayor autonomía y ocasiones de autogestión (Ortega et al., 2015).

Los jóvenes que más ocio experimentan en el centro de estudios son aquellos que prefieren una organización partida con clases todas las mañanas y todas las tardes, o jornadas más flexibles de las ofrecidas hasta el momento por las instituciones educativas. Resulta interesante destacar que los que muestran preferencias por una jornada continua solo de mañana son los que menos ocio desarrollan en las instituciones educativas.

Asimismo, otro de los hallazgos del presente estudio permite concluir que aquellos jóvenes que su jornada de clases curriculares es solo de mañanas y que, además, sienten preferencia por este tipo de jornada, practican menos ocio en el centro educativo que sus compañeros estudiantes matutinos que manifiestan preferir otro tipo de horario.

No obstante, el presente estudio, a pesar de ofrecer interesantes respuestas para (re)pensar los tiempos lectivos para transformar los centros en espacios en los que los jóvenes se encuentren identificados a través de la expresión de su verdadero ocio, debe ser valorado teniendo en cuenta que el uso de las instituciones educativas, además, está condicionado por una serie de limitaciones relacionadas con la excesiva burocratización de su estructura (Fraguela, Varela \& Caballo, 2016), con el grado de implicación y compromiso de las autoridades locales, políticas, civiles y económicas, con la escasa formación específica de los profesionales del ocio, con la reducida flexibilidad de los tiempos escolares, así como con la insuficiente disponibilidad de los espacios de las instituciones educativas, lo que dificulta el uso extracurricular de las instalaciones por problemas de seguridad, accesibilidad, recursos, equipamientos y falta de apoyo social (Gairín \& Rodríguez-Gómez, 2014).

Estos datos inspiran el interés por responder a nuevos interrogantes como los que se presentan a continuación: ¿la vinculación entre la preferencia de la jornada lectiva y el uso de los espacios educativos para la práctica del ocio se asocian, a su vez, con una tercera variable que identifica quiénes constituyen su grupo de amigos de referencia (compañeros del centro educativo $u$ otros iguales de fuera de las instalaciones educativas)?; ¿influye el tipo de actividades de ocio que se practiquen sobre el uso o no de los espacios educativos para su ocio?; ¿qué tipo de actividades de ocio son las más practicadas en los centros de educación secundaria postobligatoria?; ¿se podrían desarrollar todos los tipos de actividades de ocio en las instalaciones de un centro educativo?; ¿qué elementos clave favorecerían la promoción de la práctica de ocio en los centros de educación secundaria postobligatoria?; ¿qué factores organizativos de un centro de educación secundaria postobligatoria favorecen o dificultan la utilización o no de estos espacios como lugares de ocio? ¿están dispuestas las autoridades loca- 
les, políticas, civiles, educativas y económicas a implicarse en la revitalización de los centros educativos como espacios de referencia de identidad juvenil, social y de ocio?

La naturaleza del presente trabajo no permite responder a estas cuestiones, pero todas ellas surgen a raíz de los hallazgos de este, abriendo la puerta a nuevas investigaciones que permitan explicar y comprender la optimización de usos y aprovechamientos de las instalaciones de educación secundaria como lugares de referencia de ocio de los jóvenes españoles, de encuentro para toda la Comunidad Educativa, creando redes que favorezcan las relaciones intergeneracionales.

\section{Referencias}

Argüelles, I., Campos, A. \& López, J.A. (2015). Análisis de las actividades extraescolares en función de la variable género en el alumnado de primaria de la provincia de Granada. European Scientific Journal, 11(13), 480-498. Recuperado de http://eujournal. org/index.php/esj/ article/view/5665/5491

Caballo, M.B., Caride, J.A. \& Gradaílle, R. (2012). Entre los tiempos escolares y los tiempos de ocio: Su incidencia en la vida cotidiana de los adolescentes que cursan la Educación Secundaria Obligatoria en España. Educar em Revista, 45, 37-56. Recuperado de http://www.scielo.br/pdf/er/n45/04.pdf

Caballo, M.B., Varela, L. \& Nájera, E.M. (2017). El ocio de los jóvenes en España. Una aproximación a sus prácticas y barreras. OBETS. Revista de Ciencias Sociales, 12 (extra 1), 43-64. doi: https://doi.org/10.14198/OBETS2017.12.1.11.

Caride, J.A. (2005). A xornada escolar de sesión única en Galicia: avaliación da súa implantación e desenvolvemento nos centros de Educación Infantil, Primaria e Públicos Integrados. Santiago de Compostela: Xunta de Galicia.

Chambers, T., Pearson, A.L., Kawachi, I., Rzotkiewicz, Z., Stanley, J., Smith, M.... Signal, L. (2017). Kids in space: Measuring children's residential neighborhoods and other destinations using activity space GPS and wearable camera data. Social Science E Medicine, 193, 41-50. doi: https://doi.org/10.1016/j.socscimed.2017.09.046

Colás, P. \& Contreras, J.A. (2013). La participación de las familias en los centros de Educación Primaria. Revista de Investigación Educativa, 31 (2), 485-499. doi: http:// dx.doi.org/10.6018/rie.31.2.171031

Cruz, L. \& Morán, M.C. (2016). Jornada lectiva, aprendizajes y vida cotidiana del alumnado de Educación Secundaria Postobligatoria. Revista Interuniversitaria de Formación del Profesorado, 86 (30.2), 115-129. Recuperado de http://aufop.com/aufop/ revistas/arta/impresa/196/2012

Dewhirst, S., Pickett, K., Speller, V., Shepherd, J., Byrne, J., Almond, P., ... Roderick, P. (2014). Are trainee teachers being adequately prepared to promote the health and well-being of school children? A survey of current practice. Journal of Public Health, 36(3), 467-475. doi: http://dx.doi.org/10.1093/pubmed/fdt103

Ferreira, J.P., Pose, H. \& De Valenzuela, A.L. (2015). El ocio cotidiano de los estudiantes de Educación secundaria en España. Pedagogía Social. Revista Interuniversitaria, 25, 25-49. doi: http://dx doi.org/10.7179/PSRI_2015.25.02

Fraguela, R., Lorenzo, J.J., Merelas, T. \& Varela, L. (2013). Tiempos escolares y conciliación: análisis de familias con hijos en educación secundaria obligatoria (12-16 
años). Revista de Investigación Educativa, 31(2), 431-446. doi:http://dx.doi.org/10.6018/ rie.31.2.167001

Fraguela, R., Varela, L. \& Caballo, M.B. (2016). Percepción del profesorado de Enseñanza Secundaria Postobligatoria sobre el papel de la escuela y sus tiempos en el ocio del alumnado. Revista Interuniversitaria de Formación del Profesorado, 86 (30.2), 17-31. Recuperado de http://aufop.com/aufop/revistas/arta/impresa/196/2005

Gairín, J. \& Rodríguez-Gómez D. (2014). Leadership, Educational Development and Social Development. En I. Bogotch \& C. Shields (Eds.), International Handbook of Social [In]Justice and Educational Leadership (pp. 819-843). New York: Springer. doi:http:// dx doi.org/10.1007/978-94-007-6555-9_42

Gálvez, A. (2004). Actividad física habitual de los adolescentes de la región de Murcia. Análisis de los motivos de práctica y abandono de la actividad físico-deportiva. Murcia: Universidad de Murcia.

Gil, J.L, Felipe, J.L, Burillo, P., García-Tascón, M. \& Gallardo, L. (2010). Detection of needs in sport installation in High School: Case of province of Ávila (Spain). Journal of Sport and Health Research, 2(3), 287-304. Recuperado de http://www.journalshr. com/papers/Vol\%202_N\%203/full.pdf

Herrador, J.A. (2006). Satisfacción del profesorado de Educación Física respecto a la seguridad y conservación de las instalaciones y equipamientos deportivos en los centros educativos públicos de la provincia de Cádiz. Jaén: Servicio de publicaciones de la universidad de Jaén.

Madariaga, A. \& Romero, S. (2016). Barreras percibidas entre los jóvenes para no participar en actividades de ocio. Revista de Psicología del Deporte, 25(2), 21-26. Recuperado de https://www.rpd-online.com/article/view/v25-n4-madariaga-romero

Morales, F.J. (2013). La organización de la jornada escolar. Reflexiones sobre el estado de la cuestión para un debate necesario. Edetania, 44, 111-133. Recuperado de https:// dialnet.unirioja.es/servlet/articulo?codigo $=4596203$

Morán, M.C. \& Varela, L. (2011). Percepciones del profesorado de educación primaria sobre la conciliación de los tiempos escolares y familiares. Trabajo presentado en el XII Congreso Internacional de Teoría de la Educación (CITE), Universidad de Barcelona. Recuperado de http://www.cite2011.com/Comunicaciones/Familias/171.pdf

Morán, M.C. (2012). Incidencia de la jornada escolar en los procesos de socialización infantil. Educar em Revista, 45, 19-36. Recuperado de http://www.scielo.br/pdf/er/ n45/03.pdf

Ortega, C., Lazcano, I. \& Manuel, M. (2015). Espacios de ocio para jóvenes, de la monitorización a la autogestión. Pedagogía Social. Revista Interuniversitaria, 25, 69-89. doi: http://dx doi.org/10.7179/PSRI_2015.25.02

Ramos, R., Ponce-de-León, A. \& Sanz, E. (2010). El ocio físico-deportivo en adolescentes: Análisis y propuestas de intervención. Logroño: Universidad de La Rioja.

Ricoy, M.C. \& Fernández-Rodríguez, J. (2016). Prácticas y recursos de ocio en la adolescencia. Educatio Siglo XXI, 34(2), 103-124. doi: http://dx.doi.org/10.6018/j/263831 Sansanwal, R.M.; Derevensky, J.L.; Lupu, I.R. \& Lupu, V. (2015). Knowledge and Attitudes Regarding Adolescent Problem Gambling: A Cross-Cultural Comparative Analysis of Romanian and Canadian Teachers. International journal of mental health and addiction, 13, 33-48. doi: http://dx.doi.org/101007/s11469-014-9507-0 
Santos, M.A., Lorenzo M. \& Priegue, D. (2011). Infancia de la inmigración y educación: la visión de las familias. Revista de Investigación Educativa, 29(1), 97-110. Recuperado de revistas.um.es/rie/article/download/110351/126952

Sanz, E. (2005). La práctica físico-deportiva de tiempo libre en universitarios. Logroño: Universidad de La Rioja.

Sanz, E. \& Ponce-de-León, A. (2014). Predictores de la actividad física de tiempo libre en una población universitaria española. Revista de Pedagogía Social, 24, 183-197. doi: http://dx doi.org/10.7179/PSRI_2015.25.02

Sanz, E., Valdemoros, M.A., Ramos, R. \& Ponce-de-León, A. (2011). Claves educativas para aumentar la motivación de los jóvenes físicamente inactivos. European Journal of Education and Psychology, 4(2), 109-118. Recuperado de https://www.researchgate. net/publication/307772144_Claves_educativas_para_aumentar_la_motivacion_de_ los_jovenes_fisicamente_inactivos

Sarramona, J. \& Roca, E. (2007). La participación de las familias en la escuela como factor de calidad educativa. Participación Educativa, 4, 25-33. doi: http://dx.doi. org/10.4438/1886-5097-PE

Tinajas, A. (2012). ¿Jornada escolar continua o jornada escolar partida? Revista Iberoamericana de Educación, 59 (3), 1-10. Recuperado de http://redined.mecd.gob. es/xmlui/handle/11162/183036

Valdemoros, M. A. Sáenz-de-Jubera, M. \& Alonso, R.A. (2017). Los centros de enseñanza y la educación del ocio. Percepción de los docentes españoles según áreas geográficas. Revista Interuniversitaria de Formación del Profesorado, 89 (31.2), 103-116. Recuperado de https://dialnet.unirioja.es/servlet/articulo?codigo=6129223

Viciana, J. \& Mayorga-Vega, D. (2017). Influencing factors on planning decision-making among Spanish in-service Physical Education teachers. A population-based study. Electronic Journal of Research in Educational Psychology, 15 (3), 491-509. doi:http://dx doi.org/10.14204/ejrep.43.16112

Fecha de recepción: 26 de febrero de 2018.

Fecha de revisión: 5 de marzo de 2018.

Fecha de aceptación: 12 de octubre de 2018. 
